\title{
Urinary metabolomic fingerprinting after consumption of a probiotic strain in women with mastitis.
}

\author{
Rosa Vázquez-Fresno ${ }^{a, b}$, Rafael Llorach, ${ }^{a, b^{*}}$, , Jelena Marinic ${ }^{c}$, Sara Tulipani ${ }^{a, b, d}$, Mar Garcia- \\ Aloy $^{a, b}$, Irene Espinosa-Martos ${ }^{b, e}$, Esther Jiménez ${ }^{b, e}$, Juan Miguel Rodríguez ${ }^{b, e}$, Cristina \\ Andrés-Lacueva, ${ }^{a, b}$
}

a Biomarkers and Nutrimetabolomic Lab. Nutrition and Food Science Department, XaRTA, INSA, Torribera Campus, Pharmacy Faculty, University of Barcelona, Av. Joan XXIII s/n, 08028 Barcelona, Spain. ${ }^{b}$ INGENIO-CONSOLIDER Programme, Fun-c-food CSD2007-063, Ministry of Science and Innovation, Barcelona, Spain. ${ }^{\mathrm{c}}$ Department of Chemistry and Biochemistry, School of Medicine, University of Rijeka, Brace Brancetta 20, 51000 Rijeka, Croatia. ${ }^{\mathrm{d}}$ Biomedical Research Institute (IBIMA), Service of Endocrinology and Nutrition, Málaga Hospital Complex (Virgen de la Victoria), Campus de Teatinos s/n, University of Málaga, Málaga 29010, Spain. ${ }^{4}$ Department. of Nutrition, Food Science and Technology, Complutense University of Madrid, Avda.Puerta de Hierro, s/n, 28040 Madrid, Spain.

*Corresponding Author: Rafael Llorach.E-mail:rafallorach@ub.edu. Tel. 0034-4033798, Fax. 0034-4035931. Department of Nutrition and Food Science, XaRTA, INSA, Campus Torribera, Pharmacy School, University of Barcelona, Barcelona, Spain.

\begin{abstract}
:
Infectious mastitis is a common condition among lactating women, with staphylococci and streptococci being the main aetiological agents. In this context, some lactobacilli strains isolated from breast milk appear to be particularly effective for treating mastitis and, therefore, constitute an attractive alternative to antibiotherapy. A ${ }^{1} \mathrm{H}-\mathrm{NMR}$-based metabolomic approach was applied to detect metabolomic differences after consuming a probiotic strain (Lactobacillus salivarius PS2) in women with mastitis. Before $24 \mathrm{~h}$ urine of women with lactational mastitis was collected at baseline and after 21 days of probiotic (PB) administration. Multivariate (OSC-PLS-DA and hierarchical clustering) analysis showed metabolome differences after PB treatment. The discriminant metabolites detected at baseline of the intervention were lactose, and ibuprofen and acetaminophen (two pharmacological drugs commonly used for mastitis pain), while, after PB intake, creatine and the gut microbial co-metabolites hippurate and TMAO were detected. In addition, a voluntary desertion of the pharmacological drugs ibuprofen and acetaminophen was observed after probiotic administration. The application of NMR-based metabolomics enabled the identification of the overall effects of probiotic consumption among women suffering from mastitis and highlighted the potential of this approach in evaluating the outcomes of probiotics
\end{abstract}


consumption.To our knowledge; this is the first time that this approach has been applied in women with mastitis during lactation.

Abreviations: A, acetaminophen; CFU, colony-forming unit; FID, Free induction decay ; HCA, Hierarchical cluster analysis; IB, ibuprofen; NMR, Nuclear Magnetic Resonance; OSC-PLSDDA, Orthogonal Signal Correction Partial Least Squares-Discriminant Analysis; PB, Probiotic; TMA, Trimethylamine; TMAO, Trimethylamine-N-oxide; TSP, 3-(trimethylsilyl)-proprionate2,2,3,3-d4. Keywords: Nutrimetabolomics, metabolic biomarker, mastitis, NMR, probiotics, Lactobacillus salivarius.

\section{Introduction}

Mastitis is a common disease during lactation with an incidence of up to $33 \%$ of lactating mothers [1], and 74-95\% of cases observed in the first three months post-partum [2]. This condition is usually defined as an inflammation of the mammary gland, characterized by a variety of local and, sometimes, systemic symptoms [3]. These symptoms of mastitis are accompanied by changes in the biochemical and immunological composition of milk, and in its sensorial properties. The infectious aetiology of lactational mastitis is usually so high that some authors define the term "mastitis" as an infectious process of the mammary gland, involving staphylococci, streptococci and/or corynebacteria[2,4]. Traditionally, Staphylococcus aureus has been considered as the main aetiological agent of acute mastitis, although Staphylococcus epidermidis is emerging as the leading cause of both subacute and chronic mastitis in human medicine [5-7]. Multiresistance to antibiotics and/or formation of biofilms is very common among clinical isolates of these two staphylococcal species. This explains why this condition used to be elusive to antibiotic therapy. In addition, maternal antibiotic therapy may disturb the normal microbiota of the respiratory and digestive tracts of the mother and breastfed infant pair, a fact that may itself further inhibit defence against infection[8]. Therefore, there is a need to develop strategies that represent an alternative to the use of antibiotics and, in this context; probiotics seem to be an appealing approach. Dysbiosis is the change in the balance of microbiota composition such that it may become harmful to host health [9]. Mammary dysbiosis may lead to mastitis, a condition that represents the first medical cause for undesired weaning[3,10]. Probiotics which have been defined by the WHO/FAO as "live microorganisms that when administered in adequate amounts confer a health benefit on the consumer, can potentially influence systemic health with several mechanisms of action [11]. In this regards, probiotics in several studies are focused of research [9]. Human milk is a source of bacteria to the infant gut. In addition, modulation of maternal gut microbiota during pregnancy and lactation could have a direct effect on infant health [10]. Recent studies indicate that the mammary gland contains its own microbiota during late pregnancy and lactation. Particularly, selected strains isolated from breast milk can be good candidates for use as probiotics [12,13]. Noticeably, probiotic bacteria that are originally isolated from human milk are specifically attractive organisms since they would fulfill some of the main criteria generally recommended for human probiotics, such as human origin, a history of safe prolonged intake by a particularly sensitive population (neonates, infants), and adaptation to mucosal and dairy substrates [14].

The advent of "-omics" approaches, particularly, metabolomics analysis promises to accelerate progress in our understanding to discern the molecular pathways and biochemical mechanisms 
under the influence of the microbiota[15]. ${ }^{1} \mathrm{H}-$ Nuclear Magnetic Resonance $\left({ }^{1} \mathrm{H}-\mathrm{NMR}\right)$ based on metabolomics, combined with multivariate statistical analysis, has proven to be a powerful technology for providing profiles of numerous components present in biological fluids, enabling non-discriminant, non-destructive, highly reproducible, high-throughput analysis[16].In this regard, metabolite profiling strategies have shown a remarkable potential to differentiate and characterize infectious diseases caused by microorganisms of different strains, species and genera [17], to obtain a metabolic footprint of microorganisms [18], and to assess the effects of probiotics on the mammalian metabolism[19]. This potential prompted us to examine the applicability of NMR-based metabolomics for identifying the metabolomics changes occurring in biofluids (i.e urine) of these women as a reflect of overall biochemical effects that could occur among women suffering from lactational mastitis after probiotic consumption, an approach that has not been documented so far. In this context, a metabolomic approach based on ${ }^{1} \mathrm{H}-\mathrm{NMR}$ spectroscopy coupled with multivariate statistical analysis was used in order to investigate the metabolite composition of urine samples collected from nursing women with mastitis, before and after the oral administration of the probiotic strain Lactobacillus salivarius PS2.

\section{Material and Methods}

\subsection{Subjects and study design}

Initially, a total of 30 women with mastitis symptoms were screened and, of these, seven dropped out during the study. The reasons for dropping out, after the first analysis, were as follows: two women were suffering from severe anaemia (as assessed by blood analysis), four were taking antibiotics, and the last one moved to another country (Denmark) and could not deliver the final sample. Finally, 23 women finished the study (characteristics of the participants are reported in Suplementary Material, Table S1). All met the following criteria: breast inflammation, painful breastfeeding, milk bacterial count $>3 \log _{10} \mathrm{CFU} / \mathrm{mL}$, and milk leukocyte count $>6 \log _{10}$ cells $/ \mathrm{mL}$. None of them ingested commercial probiotic foods or supplements during the study. Women with mammary abscesses, Raynaud's syndrome or any other mammary pathology were excluded. All volunteers gave written informed consent to the protocol, which was approved by the Ethical Committee of the Hospital Clínico of Madrid (Spain). The study was registered in the ClinicalTrials.gov database (NCT00716183). All volunteers were asked to follow a controlled diet for $48 \mathrm{~h}$ (see Supplementary Material, Table S2) before the collection of the samples in order to facilitate metabolite detection in the urine samples. Women were asked to fill in a record questionnaire related to the evolution of mastitis and the presence of any potential secondary effect of probiotic treatment. The records were used to monitor adherence to the study protocol. All the 23 women reported daily ingestion of a capsule over the 21 days with the exception of two women who reported ingestion over 20 days.

The study lasted 21 days and, during this period, women consumed daily a capsule with 200 $\mathrm{mg}$ of a freeze-dried probiotic (PB) containing $\sim 9 \log _{10}$ CFU of L. SalivariusPS2. The capsules were kept at $4{ }^{\circ} \mathrm{C}$ throughout the study. Urine $(24 \mathrm{~h})$ samples from all the women were collected at the beginning (day 0, baseline) and at the end of the study (day 21, after PB) (See in Supplementary Material: Collection Procedures).

\subsection{Sample preparation}

A total of 46 urine samples (from the beginning and end of the intervention) from the 23 women were analysed. The urine samples were thawed, vortexed and centrifuged at 13,200 rpm for $5 \mathrm{~min}$. The supernatant $(600 \mu \mathrm{l})$ from each urine sample was mixed with an internal standard 
solution [120 $\mu \mathrm{L}$, consisting of $0.1 \%$ TSP (3-(trimethylsilyl)-proprionate-2,2,3,3-d4, chemical shift reference), $2 \mathrm{mM}$ of sodium azide (NaN3, bacteriostatic agent) and $1.5 \mathrm{M} \mathrm{KH}_{2} \mathrm{PO}_{4}$, in $99 \%$ deuterium water (D2O)]. The optimized $\mathrm{pH}$ of the buffer was set at 7.0, with a potassium deuteroxide solution, to minimize variations in the chemical shifts of the NMR resonances [20]. This mixture was transferred to a $5 \mathrm{~mm}$ NMR tube.

\subsection{Urine ${ }^{1} \mathrm{H}-\mathrm{NMR}$ spectra acquisition and processing}

${ }^{1} \mathrm{H}-\mathrm{NMR}$ spectra were acquired on a Varian Inova-500 $\mathrm{MHz}$ spectrometer (Varian Inc.) operating at a frequency of $500 \mathrm{MHz}$ and a temperature of $298 \mathrm{~K}$. A NOESY-presat pulse sequence was applied to suppress the residual water signal. FIDs were collected into $32 \mathrm{~K}$ data points (128 scans) with a spectral width of $14 \mathrm{ppm}$, an acquisition time of $2 \mathrm{~s}$, relaxation delay of $5 \mathrm{~s}$, and a mixing time of $100 \mathrm{~ms}[20]$. A $0.3 \mathrm{~Hz}$ line broadening function was applied to raw FIDs prior to Fourier transformation. The resulting NMR spectra were manually phased, baseline corrected and calibrated (TSP, 0.0ppm) using ACD Labs 1D NMR Processor 12.0 software (Advanced Chemistry Development Inc., Toronto, Canada). Prior to integration, each spectrum was segmented into $0.025 \mathrm{ppm}$ chemical shift bins (buckets). The residual water resonance signal $(\delta 4.73-5.00)$ was excluded, and the resulting data set in the range from 0 to 10 ppm was then submitted to MetaboAnalyst 2.0, a web-based platform for comprehensive analysis of metabolomic data (www.metaboanalyst.ca). The matrix was row-wise normalized (rows were samples) by the sum of the intensities to reduce systematic bias during sample collection.

\subsection{Multivariate statistical analysis}

The multivariate analysis was performed using the SIMCA-P+13.0 software (Umetrics, Umea, Sweden). The data set was Pareto-scaled (each variable weighted according to $1 / \sqrt{ } \mathrm{SD}$ ), and then pre-processed using Orthogonal Signal Correction (OSC) filtration before PLS-DA analysis to reduce the variability not associated with the intervention effect [21,22]. The application of this filter was by a corresponding tool provided by SIMCA-P+13.0 software. The ability to classify each individual in the correct group was assessed by $R^{2} Y$ and the prediction power of the model was assessed by the $\mathrm{Q}^{2}$ parameter. The OSC-PLS-DA model was calculated by a seven-round internal cross-validation of the data. Validation of the models and the evaluation of the degree of overfitting were then crucial to ensure that models were robust and not overfitted. For this purpose, a response permutation test $(n=200)$ was performed, and the correlation coefficient between the original $\mathrm{Y}$ and the permuted $\mathrm{Y}$ plotted against the cumulative $\mathrm{R}^{2}$ and $\mathrm{Q}^{2}$ was calculated. Generally the $\mathrm{R}^{2}$ - and $\mathrm{Q}^{2}$-intercept limits for a valid model should be less than 0.4 and 0.05 , respectively[23].

Hierarchical clustering analysis (HCA), where distance between clusters in the multivariate space is measured according to Ward's aggregation method and sorted by size, was also applied and results were plotted as a tree plot. The contribution of the signals to the separation of the classes was visualized by using S-plot, which graphically combine the weight $(p)$ of each metabolite within the model and its correlation ( $p(c o r r))$ with the modelled class designation, thus helping in the selection of statistically significant and potentially relevant metabolites. Chemical shifts showing high correlation coefficients in the S-plots $(p$ (corr) $\geq 0.5$ and ( $p$ (corr) $\leq$ 0.5 ) arbitrary cut-off, previously adopted by our research group [21,24], were included in the list of candidate markers explaining the separation between baseline and after probiotic intake, which was then submitted to the metabolite identification procedure. 


\subsection{Metabolite identification}

Discriminant metabolites were identified using the Chenomx NMR Suite 7.5 (Chenomx Inc., Edmonton, Canada) library, by comparing NMR spectral data to those available in databases such as the Human Metabolome Database (HMDB; www.hmdb.ca), the Biological Magnetic Resonance Data Bank (BMRB, www.bmrb.wisc.edu) and the Madison Metabolomics Consortium Database (MMCD, mmcd.nmrfam.wisc.edu). Metabolites from acetaminophen and ibuprofen not available in such libraries and databases are identified by bibliographic support $[25,26]$. Further, Pearson's correlation test was performed to assess correlations between signals corresponding to the same metabolite. Additionally, the Mann-Whitney test was used to test the significance for identified metabolites $(p<0.05)$.

\section{Results}

\subsection{Microbiological and clinical outcomes}

The staphylococcal/streptococcal mean count at day 0 was $3.60 \log$ colony-forming units $(\mathrm{CFU}) / \mathrm{mL}(3.34-3.85 ; 95 \% \mathrm{CI})$, while that after the probiotic treatment was $2.83 \mathrm{CFU} / \mathrm{mL}$ $(2.59-3.07)$. This difference was statistically significant ( $p$-value $<0.001$; Paired $t$ student test, $p<0.05)$. Clinically, most of the women $(>85 \%)$ that participated in the assay had improved completely or notably after the administration of the probiotic strain. The evolution of the symptoms was evaluated at day 0 (baseline) and at the end of the study by midwives from womens' health centres. At both times, the volunteers were asked to score their breast pain from 0 (extremely painful) to 10 (no pain). At the end of the study, midwives reported that 18 women had no clinical symptoms of mastitis while the remaining 5 women had improved but still displayed mild symptoms. The mean score of breast pain reported by the women at day 0 was $2.35 \pm 1.29$ (SD), while it improved in all the participants at the end of the study (8.56 \pm 2.07$)$.

\subsection{Multivariate analysis of urinary profile and metabolite identification}

A total number of 22 volunteers, after detecting and excluding one outlier spectral of a volunteer, were considered for analysis (Supplementary Material Figure S1). The OSC-PLS-DA analysis resulted in a model characterized by good robustness and predictability to explain the differences between the two sample periods, $\mathrm{R}^{2} \mathrm{Y}=0.9$ and $\mathrm{Q}^{2}=0.7$, respectively. In addition, a permutation test $(n=200)$ was carried out to validate the model. The $y$-axis intercept $\left(R^{2}\right.$ and $Q^{2}$ when the correlation coefficient is zero) is an indication of overfit. This test showed an $\mathrm{R}^{2}$ intercept of 0.34 and a $\mathrm{Q}^{2}$ intercept of -0.16 , validating the model.

Figure 1.Tree plot of sample of hierarchical clustering analysis (HCA) with Ward's aggregation

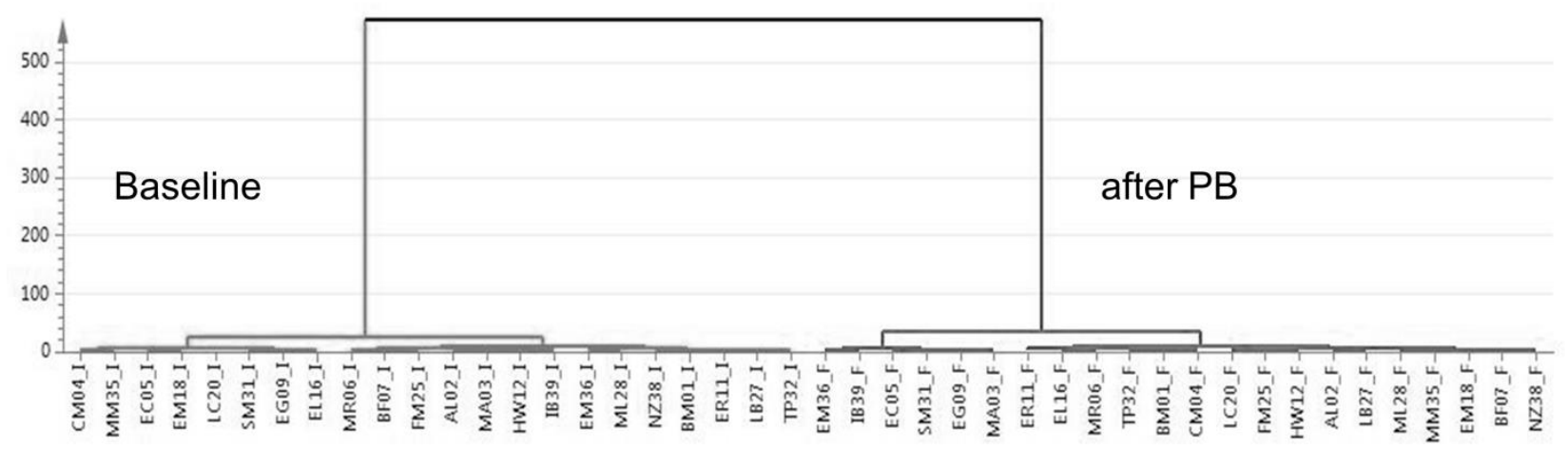


method and sorted by size. Samples of women with mastitis, at the beginning of the intervention (baseline), and after probiotic intake (after PB).

The samples were subsequently analysed using a hierarchical cluster analysis (HCA), and closer inspection revealed that the clustering of groups corresponded to before (baseline cluster) and after probiotic intake periods (Figure 1). To better visualize the contribution of signals in the separation of the classes, an S-plot was used. The markers with higher $p$ and $p$ (corr) values were the most relevant signals. The lower-left quadrant of the S-plot in Figure 2 displays the most important signals in the urine samples at baseline $(p$ (corr) $\leq-0.5)$ and the upper-right quadrant of the S-plot displays the most important signals after PB intake $p$ (corr) $\geq 0.5$ ). The signals in the middle of the figure did not show any relevance in the model. The tentative metabolites identified between both time periods with $p$ (corr) derived from the S-plot and the $p$-value from the Mann-Whitney test are shown in Table 1.

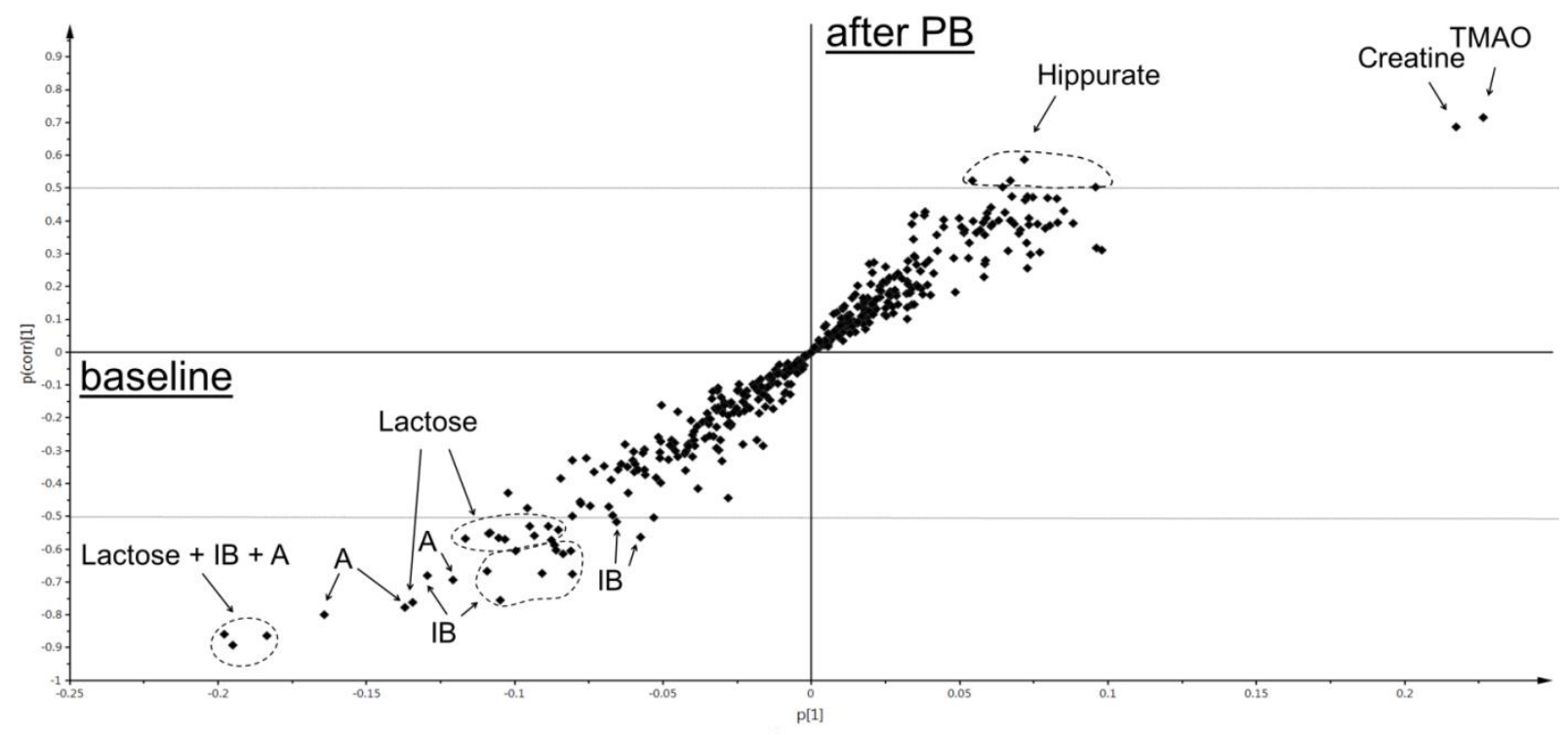

Figure 2.S-plot associated with the OSC-PLS-DA score plot obtained in women with mastitis at the beginning of the intervention and after 21 days of probiotic intake intervention. The lowerleft quadrant of the S-plot displays the discriminative chemical shifts before the 21-day probiotic intervention (baseline) and the upper-right quadrant of the S-plot displays the discriminative chemical shifts after 21 days of intervention (after PB). Dashed lines mark the threshold for each period: $p($ corr $)=+0.5$ and $p($ corr $)=-0.5$. IB: ibuprofen + ibuprofen metabolites. A: acetaminophen + acetaminophen metabolites; TMAO: Trimethylamine-N-oxide. Lactose $+\mathrm{IB}+\mathrm{A}$ indicates chemical shifts overlapped for lactose, ibuprofen and acetaminophen metabolites, achieving the greater values of the S-plot at baseline. 
Table 1.The significant metabolites derived from the S-plot in OSC-PLS-DA comparing women with mastitis at the beginning of the study (baseline) and after probiotic administration (after PB). $\uparrow$, indicates higher levels after probiotic intake ( $\mathrm{p}[\mathrm{corr}] \geq 0.5)$; $\downarrow$, indicates lower levels after probiotic intake $(\mathrm{p}[\mathrm{corr}] \leq-0.5)$. *P-value from Mann-Whitney test.

\begin{tabular}{|c|c|c|c|c|c|}
\hline Metabolites & $\begin{array}{l}\text { Chemical shift } \\
\text { (multiplicity) }\end{array}$ & After PB & Metabolic information & $\begin{array}{l}p \\
\text { (corr) }\end{array}$ & $p^{*}$ \\
\hline \multirow[t]{7}{*}{ Lactose } & $3.55(\mathrm{~m})^{1}$ & \multirow[t]{7}{*}{$\downarrow$} & \multirow{7}{*}{$\begin{array}{l}\text { Major carbohydrate present in } \\
\text { breast milk }\end{array}$} & -0.76 & $5.74 \times 10^{-7}$ \\
\hline & $3.66(\mathrm{~m})^{1}$ & & & -0.57 & $6.59 \times 10^{-5}$ \\
\hline & $3.73(\mathrm{t})^{1}$ & & & -0.55 & $8.86 \times 10^{-5}$ \\
\hline & $3.85(\mathrm{~m})^{1}$ & & & -0.57 & 0.001 \\
\hline & $4.45(\mathrm{~d}, J=7.83 \mathrm{~Hz})^{1}$ & & & -0.57 & $3.26 \times 10^{-5}$ \\
\hline & $4.67(\mathrm{~d}, J=7.83 \mathrm{~Hz})^{1}$ & & & -0.55 & 0.001 \\
\hline & $5.23(\mathrm{~d}, J=3.91 \mathrm{~Hz})^{1}$ & & & -0.53 & $6.60 \times 10^{-5}$ \\
\hline \multirow{7}{*}{$\begin{array}{l}\mathrm{IB} / \mathrm{IB} \\
\text { metabolite }\end{array}$} & $0.73(\mathrm{~d}, J=6.60 \mathrm{~Hz})^{2}$ & \multirow[t]{7}{*}{$\downarrow$} & \multirow[t]{7}{*}{ Anti-inflammatory drug } & -0.56 & 0.002 \\
\hline & $1.07(\mathrm{~d}, J=6.60 \mathrm{~Hz})^{2}$ & & & -0.68 & $1.68 \times 10^{-6}$ \\
\hline & $1.38(\mathrm{~d}, J=7.12 \mathrm{~Hz})^{2}$ & & & -0.67 & $5.16 \times 10^{-8}$ \\
\hline & $1.85(\mathrm{~m})^{2}$ & & & -0.57 & $3.00 \times 10^{-3}$ \\
\hline & $2.58(\mathrm{~d}, J=7.34 \mathrm{~Hz})^{2}$ & & & -0.68 & $3.26 \times 10^{-5}$ \\
\hline & $2.84(\mathrm{~d}, J=7.34 \mathrm{~Hz})^{2}$ & & & -0.67 & $1.73 \times 10^{-4}$ \\
\hline & $3.50(\mathrm{~s})^{2}$ & & & -0.60 & $1.90 \times 10^{-4}$ \\
\hline \multirow{2}{*}{$\begin{array}{l}\mathrm{A} / \mathrm{A} \\
\text { metabolites }\end{array}$} & $2.16(\mathrm{~s})^{3}$ & \multirow[t]{2}{*}{$\downarrow$} & \multirow[t]{2}{*}{ Analgesic drug } & -0.80 & $1.33 \times 10^{-6}$ \\
\hline & $7.13(\mathrm{~d}, J=8.80 \mathrm{~Hz})^{3}$ & & & -0.78 & $3.36 \times 10^{-6}$ \\
\hline Lactose + & $3.62(\mathrm{~m})^{\ddagger}$ & $\downarrow$ & - & -0.89 & $1.77 \times 10^{-8}$ \\
\hline $\mathrm{IB}+\mathrm{A}$ & & & & & \\
\hline
\end{tabular}

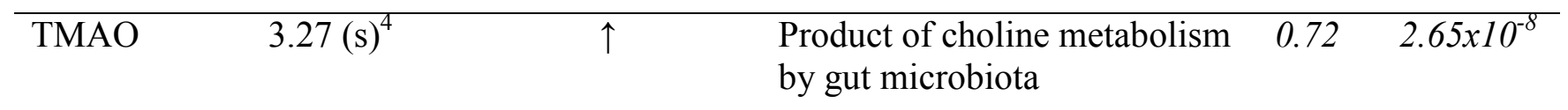

$\begin{array}{llllll}\text { Hippurate }^{\S} & 7.56(\mathrm{t}, J=7.41 \mathrm{~Hz})^{5} & \uparrow & \text { Glycine conjugate of benzoic } & 0.50 & 0.001\end{array}$




$$
\begin{aligned}
& 7.66(\mathrm{t}, J=7.41 \mathrm{~Hz})^{5} \\
& 8.54(\mathrm{br})^{5}
\end{aligned}
$$

acid associated with the

microbial degradation of

certain dietary components

(gut microbiota co-metabolite)

\begin{tabular}{llllll}
\hline Creatine $^{\S}$ & $3.03(\mathrm{~s})^{6}$ & $\uparrow$ & Energy maintenance & 0.69 & $1.74 \times 10^{-5}$ \\
\hline
\end{tabular}

Footnotes: PB: probiotic; ${ }^{*} p<0.05$, Mann-Whitney test; IB: ibuprofen; A: Acetaminophen; superscript numbers indicate correlation among the signals - same number indicates same metabolite with a correlation $r \geq 0.90 .{ }^{2}$ These signals also correlate $(r \geq 0.90)$ with other signals of IBU/IBU metabolites $(\delta 0.85, \delta 1.21, \delta 5.56, \delta 7.26, \delta 7.31) .{ }^{3}$ These signals also correlate $(r \geq 0.90)$ with other signals of $\mathrm{A}(\delta 5.10)$, TMAO: Trimethylamine-N-oxide; d:doublet, s:singlet, t:triplet.br:broad band. $J: J$-coupling value. Overlapping signal: identifications match with Chenomx library and [25]; ${ }^{1,4,5,6:}$ Identification match with Chenomx library and with standard compound from BMRB database. ${ }^{2,3}$ identification agrees with [25] and [26] and HMDB spectra. ${ }^{\S}$ The chemical shift corresponding to creatine at $\delta 3.93$, as well as hippurate at 83.96 , not shown in this table, might be affected by interference of the ibuprofen and lactose resonances. Lactose $+\mathrm{IB}+\mathrm{A}$ : chemical shifts overlapped containing lactose, ibuprofen and acetaminophen metabolites

\section{Discussion}

The baseline discriminant metabolites found in the urine of women with mastitis were lactose, ibuprofen and acetaminophen. Lactose is the major carbohydrate present in breast milk, providing a primary source of energy for infant growth. Moreover, its excretion in urine in pregnancy and lactation periods is well documented [27]. Excretion of lactose in urine is a measure of increased permeability of the lactating breast during inflammation [28]. Likewise, Fetherston et al. [28], in an attempt to identify possible predictors of mastitis in lactating mothers in the first three months post-partum, observed a significant elevation in the concentration of lactose in urine over a 24-hour period, with the highest levels observed when breast inflammation symptoms were more severe. These observations led to the conclusion that urinary excretion of lactose could be used as a reliable indicator of mastitis and a predictor of the degree and types of symptom experienced by mothers during this condition. Decreased concentration of lactose in urine following probiotic intake might, therefore, favourably affect the milk volume and the milk composition but may also represent an indicator of changes in breast physiology that allow the recovery of a mastitis mammary gland to its normal lactating state.

Higher urinary excretion of ibuprofen (IB) and acetaminophen (A) and their metabolites at baseline, and lower urinary levels after 21 days, exhibited a free desertion (without a medical prescription) of the anti-inflammatory and analgesic drugs intake in women after probiotic administration. These pharmacological drugs are widely used in the amelioration of mastitis pain [2,29]. Additionally, acetaminophen, ibuprofen and their related metabolites are readily identifiable in ${ }^{1} \mathrm{H}-\mathrm{NMR}$ urine spectra, and their spectral signatures are distinctive in human urinary metabolome after their ingestion [25,30].

After PB administration, the discriminant metabolites found were trimethylamine-N-oxide, hippurate and creatine. Trimethylamine-N-oxide (TMAO) is an oxidation product of trimethylamine, and both are products of choline metabolism. Choline from the diet is converted by gut microbiota to form trimethylamine (TMA), which is mainly oxidized to TMAO [31]. Choline requirements are very important during pregnancy and lactation. The requirements of 
choline are also likely to increase during pregnancy and lactation because large amounts of choline must be delivered to the foetus across the placenta [32] and secreted into breast milk [33] from maternal circulation. The increased levels of TMAO in urine could suggest a beneficial probiotic effect in the choline metabolism of the lactating women from the present study. Additionally, the presence of TMAO in human urine may also be related with high meat [34] and fish [35] intake.

Many factors have been found to be associated with changes in the urinary excretion of hippurate (a metabolite strongly associated with diet and intestinal microbiota) [36]. To our knowledge, though, no studies evaluating women with mastitis have found a link with hippurate; however, a study of cows' milk metabolites found lower levels of this metabolite in mastitis cows[37]. Thus, an increase in the levels of this metabolite after probiotic intake in urine could be reflected by increased levels of hippurate in milk, in consonance with an improvement in the health of women from the present study.

Several studies have demonstrated the importance of gut microbiota in contributing to the excretion of metabolites such as TMAO and hippurate. For instance, their excretion was not observed in germ-free animals; however, after the exposure to intestinal microbiota, hippurate and TMAO excretion was detected [38]. Moreover, some studies reported a reduction in the urinary excretion of both hippurate and TMAO in rats treated with antibiotics [39,40]. In addition, this fact supports the non-antibiotic intake from the participants during the study. In this regard, research is ongoing concerning the important effects of gut microbiota on human health $[41,42]$.

Finally, the creatine pool is important for maintaining ATP levels when energy demand is transiently greater than the rate of ATP synthesis [43]. Under physiological conditions, creatine is spontaneously converted to creatinine, which is subsequently excreted in urine [44]. However, elevated urinary levels of creatine and creatinine are also used as biomarkers of protein intake, associated with meat consumption [45].

A recent study reported a higher excretion of creatine in the breastfeeding stage, which indicates the ability of breastfeeding women to adjust to these metabolic demands imposed by breastfeeding [46]. The increased urinary level of creatine found after probiotic intervention could exhibit an increased endogenous synthesis of creatine and may reflect a re-establishment of the normal lactation stage in these women after the intervention.

A possible lack of the present study is a proper control group of mastitis women without the administration of the probiotic. However, the objective of this study is to assess the urinary metabolomics changes in mastitis women before and after the probiotic administration. The amelioration of mastitis symptoms was previously tested in two randomly selected groups of women with mastitis: one group consuming probiotic and the other without probiotic administration (control group)[13], concluding that the probiotic administration appear to be an efficient alternative for the treatment of lactational infectious mastitis during lactation.

\section{Conclusions}

Characterization of the urine metabolic profile of the nursing women with mastitis at the end of the intervention by an NMR-based metabolomic approach showed that probiotic supplementation decreased lactose excretion which could suggesta normalization of breast permeability. Additional studies, examining the correlation of lactose with inflammatory and immunological mediators, along with the mother's health status, are needed to confirm this possibility. 
The reduction in the urine excretion of ibuprofen and acetaminophen demonstrates the voluntary desertion of anti-inflammatory and analgesic drugs intake in these women at the end of the study. Moreover, an increase of TMAO, creatine and hippurate might reflect changes in choline metabolism, in energetic pathways and in gut microbiota metabolism, in line with a reported improvement in the health status of the women at the end of the intervention.

By profiling urinary metabolites we have demonstrated the applicability of NMR-based metabolomics for identifying the overall biochemical effects of oral probiotic administration to women suffering from lactational mastitis, and highlighted the potential of this approach in evaluating the outcomes of probiotics consumption.

\section{Conflict of interest}

The authors have declared no conflict of interest.

\section{Acknowledgments}

We thank the participants for their collaboration in the study. This research was supported by Spanish National Grants from the Spanish Ministry of Science and Innovation (MICINN): CICYT-AGL 2006-14228-C03-02, AGL2009-13906-C02-01, AGL2010-10084-E , AGL201015420 and CONSOLIDER INGENIO 2010 Programme, FUN-C-FOOD (CSD2007-063), and a research contract from the Nutricia/Danone Research Centre for Specialised Nutrition (Utrecht, The Netherlands). RVF, ST and RLl would like to thank the FPI 2010 fellowship, the "Juan de la Cierva" and "Ramón y Cajal" programmes respectively from MICINN and the European Social Funds. MGA and MJ would like to thank the predoctoral fellowships awarded by the Generalitat de Catalunya (FI-DRG-2011)and the Faculty of Medicine, University of Rijeka TransMedRi 256686) and EUFP7-REGPOT- 2010-5 grants, respectively.

\section{Appendix A. Supplementary data}

Supplementary data associated with this article can be found, in the online version.

\section{References}

[1] Foxman B, D'Arcy H, Gillespie B, Bobo JK, Schwartz K. Lactation mastitis: occurrence and medical management among 946 breastfeeding women in the United States. Am J Epidemiol 2002;155:103-14.

[2] WHO. Mastitis: Causes and management. 2000.

[3] Lawrence RL, RM. Breastfeeding. A guide for the medical profession., 6th ed. St. Luis: Elsevier Mosby, 2005.

[4] Barbosa-Cesnik C, Schwartz K, Foxman B. Lactation mastitis. JAMA 2003;289:1609-12.

[5] Delgado S, Arroyo R, Martín R, Rodríguez JM. PCR-DGGE assessment of the bacterial diversity of breast milk in women with lactational infectious mastitis. BMC Infect Dis 2008;8:51. 
[6] Delgado S, Arroyo R, Jiménez E, Marín ML, del Campo R, Fernández L, et al. Staphylococcus epidermidis strains isolated from breast milk of women suffering infectious mastitis: Potential virulence traits and resistance to antibiotics. BMC Microbiol 2009;9:82.

[7] Cusack L, Brennan M. Lactational mastitis and breast abscess - diagnosis and management in general practice. Aust Fam Physician 2011;40:976-9.

[8] Kummeling I, Stelma FF, Dagnelie PC, Snijders BE, Penders J, Huber M, et al. Early life exposure to antibiotics and the subsequent development of eczema, wheeze, and allergic sensitization in the first 2 years of life: The koala birth cohort study. Pediatrics 2007;119:e225-31.

[9] Walker AW, Lawley TD. Therapeutic modulation of intestinal dysbiosis. Pharmacol Res 2013;69:75-86.

[10] Fernández L, Langa S, Martín V, Maldonado A, Jiménez E, Martín R, et al. The human milk microbiota: Origin and potential roles in health and disease. Pharmacol Res 2013;69:1-10.

[11] Lee IC, Tomita S, Kleerebezem M, Bron PA. The quest for probiotic effector moleculesunraveling strain specificity at the molecular level. Pharmacol Res 2013;69:61-74.

[12] Arroyo R, Martin V, Maldonado A, Jimenez E, Fernandez L, Rodriguez JM. Treatment of infectious mastitis during lactation: Antibiotics versus oral administration of lactobacilli isolated from breast milk. Clin Infect Dis 2010;50:1551-8.

[13] Jiménez E, Fernández L, Maldonado A, Martín R, Olivares M, Xaus J, et al. Oral administration of lactobacillus strains isolated from breast milk as an alternative for the treatment of infectious mastitis during lactation. Appl Environ Microbiol 2008;74:4650-5.

[14] Collins JK, Thornton G, Sullivan GO. Selection of probiotic strains for human applications. Int Dairy J 1998;8:487-90.

[15] Martin F-PJ, Collino S, Rezzi S. 1H NMR-based metabonomic applications to decipher gut microbial metabolic influence on mammalian health. Magn Reson Chem 2011;49:S47-S54.

[16] Lenz EM, Wilson ID. Analytical strategies in metabonomics. J Proteome Res 2007;6:44358.

[17] Slupsky CM, Cheypesh A, Chao DV, Fu H, Rankin KN, Thomas J. M, et al. Streptococcus pneumoniae and staphylococcus aureus pneumonia induce distinct metabolic responses. $\mathbf{J}$ Proteome Res 2009;8:3029-36.

[18] Sulek K, Frandsen H, Smedsgaard J, Skov T, Wilcks A, Licht T. Metabolic footprint of lactobacillus acidophilus ncfm at different pH. Metabolomics 2012;8:244-52.

[19] Martin FP, Wang Y, Sprenger N, Yap IK, Lundstedt T, Lek P, et al. Probiotic modulation of symbiotic gut microbial-host metabolic interactions in a humanized microbiome mouse model. Mol Syst Biol 2008;4:157.

[20] Vazquez-Fresno R, Llorach R, Alcaro F, Rodriguez MA, Vinaixa M, Chiva-Blanch G, et al. (1)H-NMR-based metabolomic analysis of the effect of moderate wine consumption on subjects with cardiovascular risk factors. Electrophoresis 2012;33:2345-54.

[21] Tulipani S, Llorach R, Jáuregui O, López-Uriarte P, Garcia-Aloy M, Bullo M, et al. Metabolomics unveils urinary changes in subjects with metabolic syndrome following 12week nut consumption. J Proteome Res 2011;10:5047-58.

[22] Pujos-Guillot E, Hubert J, Martin J-F, Lyan B, Quintana M, Claude S, et al. Mass spectrometry-based metabolomics for the discovery of biomarkers of fruit and vegetable intake: Citrus fruit as a case study. J Proteome Res 2013;12:1645-59. 
[23] Van Der Voet H. Comparing the predictive accuracy of models using a simple randomization test. Chemometr Intell Lab Syst 1994;25:313-23.

[24] Llorach R, Urpi-Sarda M, Jauregui O, Monagas M, Andres-Lacueva C. An LC-MS-based metabolomics approach for exploring urinary metabolome modifications after cocoa consumption. J Proteome Res 2009;8:5060-8.

[25] Holmes E, Loo RL, Cloarec O, Coen M, Tang H, Maibaum E, et al. Detection of urinary drug metabolite (xenometabolome) signatures in molecular epidemiology studies via statistical total correlation (NMR) spectroscopy. Anal Chem 2007;79:2629-40.

[26] Neunzig I, Göhring A, Drăgan C-A, Zapp J, Peters FT, Maurer HH, et al. Production and NMR analysis of the human ibuprofen metabolite 3-hydroxyibuprofen. J Biotechnol 2012;157:417-20.

[27] Date JW. The excretion of lactose and some monosaccharides during pregnancy and lactation. Scand J Clin Lab Invest 1964;16:589-96.

[28] Fetherston CM, Lai CT, Hartmann PE. Relationships between symptoms and changes in breast physiology during lactation mastitis. Breastfeed Med 2006;1:136-45.

[29] Strong GD. Provider management and support for breastfeeding pain. J Obstet Gynecol Neonatal Nurs 2011;40:753-64.

[30] Loo RL, Coen M, Ebbels T, Cloarec O, Maibaum E, Bictash M, et al. Metabolic profiling and population screening of analgesic usage in nuclear magnetic resonance spectroscopybased large-scale epidemiologic studies. Anal Chem 2009;81:5119-29.

[31] Russell WR, Hoyles L, Flint HJ, Dumas M-E. Colonic bacterial metabolites and human health. Curr Opin Microbiol 2013;16:246-54.

[32] Welsch F. Studies on accumulation and metabolic fate of (N-Me3h)choline in human term placenta fragments. Biochem Pharmacol 1976;25:1021-30.

[33] Holmes-McNary MQ, Cheng WL, Mar MH, Fussell S, Zeisel SH. Choline and choline esters in human and rat milk and in infant formulas. Am J Clin Nutr 1996;64:572-6.

[34] Stella C, Beckwith-Hall B, Cloarec O, Holmes E, Lindon JC, Powell J, et al. Susceptibility of human metabolic phenotypes to dietary modulation. J Proteome Res 2006;5:2780-8.

[35] Svensson BG, Åkesson B, Nilsson A, Paulsson K. Urinary excretion of methylamines in men with varying intake of fish from the baltic sea. J Toxicol Environ Health 1994;41:41120.

[36] Lees HJ, Swann JR, Wilson ID, Nicholson JK, Holmes E. Hippurate: The natural history of a mammalian-microbial cometabolite. J Proteome Res 2013;12:1527-46.

[37] Sundekilde UK, Poulsen NA, Larsen LB, Bertram HC. Nuclear magnetic resonance metabonomics reveals strong association between milk metabolites and somatic cell count in bovine milk. J Dairy Sci 2013;96:290-9.

[38] Nicholls AW, Mortishire-Smith RJ, Nicholson JK. NMR spectroscopic-based metabonomic studies of urinary metabolite variation in acclimatizing germ-free rats. Chem Res Toxicol 2003;16:1395-404.

[39] Swann JR, Tuohy KM, Lindfors P, Brown DT, Gibson GR, Wilson ID, et al. Variation in antibiotic-induced microbial recolonization impacts on the host metabolic phenotypes of rats. J Proteome Res 2011;10:3590-603.

[40] Yap IKS, Li JV, Saric J, Martin F-P, Davies H, Wang Y, et al. Metabonomic and microbiological analysis of the dynamic effect of vancomycin-induced gut microbiota modification in the mouse. J Proteome Res 2008;7:3718-28. 
[41] Nicholson JK, Holmes E, Wilson ID. Gut microorganisms, mammalian metabolism and personalized health care. Nat Rev Microbiol 2005;3:431-8.

[42] Leser TD, Mølbak L. Better living through microbial action: The benefits of the mammalian gastrointestinal microbiota on the host. Environ Microbiol 2009;11:2194-206.

[43] da Silva RP, Nissim I, Brosnan ME, Brosnan JT. Creatine synthesis: Hepatic metabolism of guanidinoacetate and creatine in the rat in vitro and in vivo. Am J Physiol Endocrinol Metab 2009;296(2): E256-E261.

[44] Wyss M, Kaddurah-Daouk R. Creatine and creatinine metabolism. Physiol Rev 2000;80:1107-213.

[45] Rasmussen LG, Winning H, Savorani F, Toft H, Larsen TM, Dragsted LO, et al. Assessment of the effect of high or low protein diet on the human urine metabolome as measured by nmr. Nutrients 2012;4:112-31.

[46] Sachse D, Bærug A, Sletner L, I. Birkeland K, Nakstad B, Jenum AK, et al. Urine NMR metabolomics analysis of breastfeeding biomarkers during and after pregnancy in a large prospective cohort study. Scand J Clin Lab Invest 2014:1-9. 